\title{
La qualité: une interaction de toutes les forces en présence dans le domaine médical
}

\author{
Michael Peltenburga , Hans Kernen ${ }^{b}$, Philippe Schneiderc, Georg von Below ${ }^{\prime}$, Gertrud Waldis ${ }^{e}$, Hans Anton Vogel ${ }^{e}$, Jean-Claude Rochese, \\ Werner Schoop ${ }^{e}$
}

Deutsch erschienen

in Nr. 18/2005.

a Dr méd., responsable GT Q du VEDAG

b Conseiller en organisation, Kernen SA, Küsnacht/Zurich (accompagnement de processus)

c Enseignant et directeur du centre de compétences en gestion de la qualité, Berner Fachhochschule HSA, Berne

d Dr méd., respondable du domaine DDQ, FMH

e Drs méd., membres du groupe de travail «Qualité»

1 Interaction de toutes les personnes concernées en vue d'un résultat efficace.

Correspondance:

Dr Michael Peltenburg

Praxis Post

CH-8340 Hinwil

E-mail: fmhqualitaet@hin.ch
La qualité de la prestation médicale est devenue une notion-clé de la médecine actuelle. Cependant, il n'existe aucune définition généralement applicable de la qualité pour ce type de prestation. Le terme de qualité a subi d'importantes modifications au cours de la dernière décennie et les spécificités des «co-productions humaines» [1] ne sont définies et expliquées que de manière imprécise. D'autre part, les systèmes de gestion de la qualité applicables dans l'industrie, tels que les certifications ISO, ne sont guère utiles dans le domaine médical. Quelle évolution l'exigence de qualité a-t-elle subie? Quelles sont les conséquences de cette évolution?

Auparavant, on entendait par qualité une prestation globale, notion qui incluait la quantité, le type, les caractéristiques, le fournisseur et le prix de la prestation. De même que l'on considérait une montre suisse comme un bon produit, les médecins affiliés à la FMH passaient pour de bons médecins. En règle générale, les fournisseurs de prestations définissaient eux-mêmes la qualité et les destinataires se contentaient du produit reçu sans établir de distinction entre qualité visée, qualité négociée ou qualité dépassant les attentes.

Aujourd'hui, la qualité est négociée. Elle est le fruit de discussions entre les fournisseurs de prestations (prestataires) et les bénéficiaires de ces prestations (ou leurs représentants et/ou leurs proches), ainsi que les politiques, les caisses-maladie, les économistes, etc. Il ne s'agit plus de choisir la meilleure qualité possible ni la plus chère (en fonction de l'offre), mais de proposer une qualité sur mesure.

\section{Notion de qualité en mutation}

La notion de qualité globale comme on l'entendait autrefois est désormais fragmentée en prestation professionnelle et en prestation économique. Le caractère économique d'une prestation pouvant être vérifié d'après des indicateurs de gestion commerciale, l'accent est principalement mis sur cet aspect-là malgré une pertinence controversée. Les différends entre médecins et assureurs portent principalement sur ces indica- teurs d'économie d'entreprise (p. ex. l'évaluation de la valeur moyenne) et ne concernent que rarement la qualité technique ou humaine.

Les prestations axées sur des personnes comprennent une qualité logistique et technocratique, une qualité humaine et une qualité professionnelle allant dans le sens d'une co-production. La qualité logistique est liée entre autres à des facteurs externes, dont font partie les laboratoires, les instituts de radiologie, etc., ainsi que les instances et procédures de contrôle. Vient s'y ajouter la qualité du service fourni, qui se mesure notamment en termes de joignabilité et d'amabilité du prestataire. Ces différents aspects se

Les «Lignes directrices pour la qualité de la médecine» visent une gestion globale de la qualité médicale

- La qualité est une dynamique modifiable et sans valeur fixe.

- La qualité fait partie d'un système «apprenant».

- La qualité est une co-production.

- La qualité visée est négociée et obtenue en collaboration avec les groupes concernés.

- La qualité nécessite une amélioration constante et met l'accent sur le développement et le soutien.

- La qualité peut être prouvée et décrite.

- De nombreux aspects qualitatifs ne peuvent pas être quantifiés et doivent être redéfinis en permanence.

Les lignes directrices décrivent

- La qualité dans sa complexité.

- Les conceptions du corps médical en matière de qualité.

- Les critères - dûment motivés - définissant les instruments de qualité.

- Les arguments sur lesquels fonder les négociations avec les co-producteurs.

En offrant une structure et un soutien, les lignes directrices constituent la base nécessaire pour diriger la qualité médicale. 
répercutent sur la satisfaction subjective du patient. La qualité professionnelle repose quant à elle avant tout sur la co-production, autrement dit sur la relation complexe et la dépendance entre médecin et patient. C'est ici la qualité de la prestation qui prime, de même que les normes techniques. Il s'agit de prouver que les effets visés ont été obtenus, d'encourager, de garantir et/ou d'améliorer ces effets.

\section{Questions centrales}

Comment fournir une bonne prestation médicale? Comment en promouvoir et garantir la qualité? Quelles sont les réponses appropriées à nos problèmes?

La qualité, qui résulte de la combinaison de connaissances d'experts (recherche), d'un savoir-faire et d'influences subjectives, est une notion essentielle tant dans le domaine social et pédagogique que médical. Les spécialistes et toutes les personnes directement concernées y sont confrontés. Ils fixent les objectifs et les indicateurs adéquats et établissent des normes contraignantes: une tâche exigeante à tous points de vue. Il ne suffit pas pour ce faire de convaincre ses propres rangs (de la nécessité d'une gestion des erreurs, par exemple), mais également les partenaires et les groupes d'intérêts, qu'il convient de motiver en vue de définir une conception globale de la qualité tout en procédant aux limitations mutuelles qui s'imposent. Dans ce processus, les lignes directrices pour la qualité de la médecine forment la base d'une discussion constructive sur la qualité.

\section{Historique des lignes directrices pour la qualité de la médecine}

En automne 2003, le comité du VEDAG (Verband deutschschweizerischer Ärztegesellschaften) a invité les présidents et présidentes de ses sociétés cantonales de médecine, ainsi que leurs délégués et déléguées à la qualité, à une séance de réflexion sur le thème de la qualité médicale. Le groupe de travail «Qualité» * a été créé à la suite de cette réunion. Il a pour tâche d'élaborer les bases nécessaires à une gestion globale de la qualité.

En janvier 2004, le groupe de travail «Qualité» a demandé à différentes organisations professionnelles, sociétés de discipline médicale, assurances et partis politiques quels étaient les concepts, instruments et projets ayant cours dans leurs rangs en matière de qualité. Il est ré-

\section{Lignes directrices pour la qualité} de la médecine

- Principes directeurs des médecins suisses

- Conceptions du corps médical en matière de qualité

- Définition de la qualité en termes de résultats

- Processus de développement de la qualité

- Structures permettant de s'orienter

- Promotion, développement et assurance de la qualité

- Activités de relations publiques

sulté de cette enquête qu'on ne trouve quasiment aucune aide pour s'orienter en ce qui concerne la qualité dans le domaine de la santé. En conséquence, le groupe de travail a tout d'abord résumé sa prise de position dans un article [2] pour ensuite fixer des objectifs prioritaires et définir de nouvelles notions. Le but visé était de créer un instrument destiné aux médecins, de lancer le débat et de réunir tous les protagonistes du système de santé autour d'une même enseigne et d'une vision commune de la qualité. C'est ainsi que les lignes directrices pour la qualité de la médecine présentées ci-après ont vu le jour.

\section{Lignes directrices pour la qualité de la médecine}

Les présentes lignes directrices constituent le fondement d'une gestion globale de la qualité. Elles sont axées sur la co-production, autrement dit sur une interaction de toutes les forces en présence axée sur le résultat. Les lignes directrices montrent les différents aspects de la qualité. Elles nous aident à mieux situer notre action et à fixer les bonnes priorités dans nos rapports avec autrui.

\section{Principes directeurs de l'action médicale}

- Nous, médecins suisses, nous engageons pour l'être humain en vue de soulager ses maux et de promouvoir sa santé. Le respect, un encadrement digne de l'être humain ainsi que les traitements et soins nécessaires sont offerts à tous les patients, indépendamment de leurs particularités et même s'ils se situent hors des normes sociales. Nous ne faisons preuve d'aucune tolérance à l'égard des abus dans la relation médecin-patient.

- Notre travail est une co-production [3]. Autrement dit, nous nous engageons, en collaboration avec différents partenaires du domaine de la santé, à maintenir la santé du patient et
3 Prestation de qualité obtenue avec différents partenaires. 


\section{Lignes directrices}

- Nous, médecins suisses, nous engageons pour l'être humain en vue de soulager ses maux et de promouvoir sa santé.

- Notre travail est une co-production.

- Nous travaillons en partenariat avec nos co-producteurs.

- Nous travaillons selon les principes de la médecine factuelle, de manière transparente et axée sur le patient.

- Nous travaillons efficacement, de manière économique et sans charger inutilement le patient.

- Nous tenons compte des exigences sociales, politiques, éthiques, économiques et de celles liées aux assurances.

- Nous encourageons l'introduction de mesures correctrices et la coopération et nous efforçons de reconnaître nos limites.

- Nous nous formons en permanence, travaillons de manière réfléchie, faisons preuve de créativité et d'engagement.

à soulager ses maux. Nous soutenons le patient dans l'acceptation de ses handicaps et l'accompagnons jusqu'à son décès.

- Nous travaillons en partenariat avec nos co-producteurs [4]. Nous sommes en contact permanent avec nos confrères, les institutions sociales, les organisations de patients, les assureurs et les politiques pour le bien du patient.

- Nous travaillons selon les principes de la médecine factuelle, de manière transparente et axée sur le patient. Nous voulons comprendre le patient dans son propre environnement et nous l'écoutons. En faisant preuve d'ouverture et d'honnêteté, nous instituons une relation de confiance basée sur la continuité. Nous prenons nos décisions d'un commun accord avec le patient. Nous respectons ses décisions et ses actions et assumons la responsabilité de nos actes. Nous travaillons dans la transparence, clarifions le mandat de traitement, donnons des informations compréhensibles et adoptons une attitude ouverte face aux événement critiques et à l'erreur.

- Nous travaillons efficacement, de manière économique et sans charger inutilement le patient. Nous acceptons le devoir incombant aux assureurs de contrôler la qualité de même que les conditions économiques générales.

4 Patients, assureurs, médecins, organisations de soins à domicile, etc. et de la diversité culturelle et tenons compte de la situation actuelle dans notre travail. Nous prenons au sérieux les exigences du monde politique ainsi que le mandat qui nous a été confié en matière de qualité. Nous répondons du respect des exigences éthiques.

- Nous encourageons l'introduction de systèmes de retour d'information correctifs et acceptons pour ce faire les avis externes. Nous sommes conscients de nos limites, les acceptons et en faisons part aux autres. Nous coopérons lors des transferts, du travail en équipe et des soins interdisciplinaires.

- Nous nous formons en permanence, travaillons de manière réfléchie, faisons preuve de créativité et d'engagement.

- Nous nous engageons activement dans la formation postgraduée et continue et coopérons dans les questions qui ne peuvent être résolues uniquement par la médecine.

\section{Conceptions du corps médical en matière} de qualité

La qualité est avant tout marquée par l'intégrité personnelle du médecin dans son rapport avec le patient. Cet aspect a jusqu'ici malheureusement trop peu été pris en compte dans les études de médecine, mais demeure l'essence de la qualité médicale, à savoir une relation vivante, authentique et fructueuse entre médecin et patient, favorisée par un style de communication ouvert et constructif reposant sur la confiance et l'empathie.

Notre représentation des objectifs de l'activité médicale, ainsi que les rôles du médecin et du patient doivent constamment être remis en question et nourrissent nos réflexions. Par l'autocritique, nous aboutissons à une qualité optimale. L'auto-réflexion et les réflexions de tiers nous accompagnent en permanence dans notre activité et sont des outils de travail essentiels.

L'autonomie décisionnelle est une des qualités caractéristiques de notre activité. Elle seule nous permet d'œuvrer en toute conscience, de définir clairement les limites entre ce qui est possible et ce qui ne l'est pas et de soutenir nos «coproducteurs» dans leur activité. Les exigences qualitatives que cela suppose nous permettent de répondre aux attentes.

\section{Qualité des résultats}

La qualité du résultat final d'une «co-production» se définit par rapport aux attentes, aux ressources et aux investissements. Elle demande à être clarifiée d'emblée et est donc négociée et fixée d'entente avec les co-producteurs. Les indicateurs possibles en sont: la santé (prix de la gué- 
rison, degré d'invalidité, qualité du décès), la qualité de vie recouvrée ou perdue, la capacité de travail ou la satisfaction des «co-producteurs».

\section{Processus de développement de la qualité}

De quelle manière fournissons-nous nos prestations afin de parvenir à la qualité convenue? Le développement de la qualité résulte de processus se déroulant à différents niveaux. Cette évolution n'est pas limitée dans le temps, ses priorités sont changeantes et sa dynamique l'est aussi.

Le développement individuel de la qualité comprend notre formation continue personnelle et professionnelle ainsi que l'auto-évaluation, l'optimalisation ciblée de notre pratique médicale et une relation équilibrée entre travail, vie privée et loisirs («Housekeeping»).

Le développement de la qualité axé sur la pratique comporte des améliorations internes telles que la standardisation des processus de travail courants, laquelle permet à son tour de procéder à de nouveaux ajustements. En nous exerçant régulièrement à maîtriser des situations d'urgence, nous sommes préparés aux incidents. Les conflits entre personnes sont la principale cause de problèmes au cabinet médical. Tout ce qui mène à l'instauration d'une relation de confiance entre les participants en vue d'une «co-production» réussie s'inscrit dans cette perspective: collégialité, intonation agréable avec les autres membres du cabinet médical, ouverture en matière de communication avec les collègues, les patients et les partenaires.

Par développement de la qualité contextuelle, on entend le développement spécifique de la qualité médicale. L'appréciation constante de soi et de son travail par le biais de l'auto-critique, la comparaison avec les autres (entre pairs) et l'évaluation par des tiers sont des étapes vers ce but. Un autre instrument de développement de la qualité consiste à collaborer avec des spécialistes et des professionnels sur la base de guides de pratique médicale ou du travail en équipe.

Toute personne qui travaille se trompe parfois. Jusqu'à présent, le corps médical n'était pas familier de l'analyse systématique des événements critques ou des erreurs. Il faut apprendre à gérer les situations critiques. Seule une gestion transparente et rigoureuse des événement critiques peut devenir source d'apprentissage. Ce n'est que lorsqu'un tel processus ne fonctionne plus qu'il faut procéder à des jugements et des sanctions. L'analyse des fautes commises nous permet non seulement de découvrir nos propres manquements, mais également les erreurs inhérentes ou externes au système qu'il est ensuite possible de corriger par des mesures appropriées.
Des structures pour aider à s'orienter

Les structures peuvent être soit limitatives, soit apporter soutien, sécurité et orientation. Lorsqu'elles servent de moyen auxiliaire pour atteindre la qualité voulue, elles ne sauraient en aucun cas représenter une fin en soi.

Les structures internes peuvent être établies ou modifiées plus ou moins librement. Une infrastructure technique et des locaux appropriés nous permettent de travailler de manière ciblée. En organisant les structures administratives le plus clairement et le plus simplement possible, nous pouvons garantir le déroulement sans accrocs des processus de travail. La tenue correcte, honnête et juridiquement nécessaire du dossier du patient est désormais une priorité qui demande beaucoup de temps. Il s'agit à cet égard de trouver la mesure raisonnable. Une gestion du temps souple et appropriée offre non seulement une plus grande efficacité sur le plan économique, elle est également favorable au client et garantit que le médecin soit facilement joignable et que sa suppléance soit bien organisée. Une structure du personnel adaptée aux besoins se répercute favorablement sur le climat de travail au sein de l'institution ainsi que sur les prestations envers tous les partenaires. Des structures judicieuses nous permettent non seulement de profiter de notre famille et de nos loisirs, mais également d'avoir du temps pour d'autres intérêts.

Les structures définies par des tiers sont souvent complexes et nous n'avons guère la possibilité de les modifier. Travailler en réseau signifie rechercher le dialogue avec les patients, les médecins, les hôpitaux, les thérapeutes, les assureurs, les juristes, les instances officielles et autres partenaires. L'impossibilité d'agir au niveau des structures fait de l'ouverture au dialogue un défi permanent, qu'il ne faut pas sous-estimer. Il nous est possible d'exercer une légère influence sur les offres de formation postgraduée et continue dans les cliniques et les universités, mais en règle générale nous dépendons de l'offre existante. Les conditions financières générales sont en grande partie dictées par des facteurs externes, tant économiques que politiques. Comme il s'agit là d'un élément-clé d'une bonne prise en charge médicale, nous sommes invités, en tant que médecins, à participer activement aux décisions politiques et sociales.

\section{Promotion, développement et assurance de la qualité}

La qualité, qui s'adapte aux nouvelles connaissances et aux nouvelles formes de vie, est en constante évolution. La bureaucratie et des nor- 
mes définies risquent de freiner son évolution. Nous devons accepter et discuter les annonces d'incidents critiques («critical incident reporting» ou «CIR»), les critiques et les propositions des cabinets médicaux et des hôpitaux, des patients, des autorités et des assureurs, car elles nous permettront en fin de compte d'obtenir les améliorations escomptées.

Les directives et les normes sont utiles pour décrire la qualité telle qu'elle a été négociée, et ce pour toutes les prestations convenues avec nos co-producteurs. La qualité des prestations est ainsi fournie de manière à pouvoir être évaluée par nous-mêmes et par nos partenaires. Cette transparence nous donne de la crédibilité. Lorsque une lacune qualitative est constatée, nous recherchons tout d'abord le dialogue et faisons des suggestions concrètes d'amélioration. Si celles et ceux qui ont généré le problème ne proposent par de solutions et/ou si les mesures prises sont insuffisantes, une procédure juridique sera engagée avec les conséquences que cela implique (retrait des certificats, exclusion et suppression de l'autorisation de pratiquer). Ces sanctions doivent également être conçues de manière transparente, de sorte qu'elles puissent être comprises par tous.

\section{Activités de relations publiques}

Etant donné que la qualité est générée en commun (co-production), nous informerons la population, d'entente avec nos partenaires, via la presse et l'internet. Nos efforts en matière de qualité sont convaincants et rendus publics. Cela présuppose que nous fassions preuve d'ouver- ture face à la critique, aux remarques et aux suggestions d'amélioration. Il est nécessaire que le sigle FMH devienne un label de qualité, et ce également en vue de la reconnaissance de notre profession sur le plan européen.

Si le travail de conception des présentes lignes directrices est terminé, celles-ci seront toutefois constamment adaptées et remaniées par les coproducteurs dès leur introduction.

\section{Perspectives}

Les présentes lignes directrices ont été présentées le 21 avril 2005 au Dr Jacques de Haller, président de la FMH, au Dr L. T. Heuss, au Comité central de la FMH ainsi qu'aux présidents et présidentes des sociétés représentées à la Chambre médicale. Ce document est à la base de l'actuel débat sur la qualité et sera soumis en tant que «travail en cours» à la Chambre médicale du 19 mai 2005 pour approbation.

Le groupe de travail «Qualité» prévoit une séance d'information avec les «co-producteurs» en novembre 2005.

\section{Outils d'information et de diffusion}

Les «Lignes directrices pour la qualité de la médecine» se trouvent comme mindmap sur internet: www.saez.ch/mindmap.pdf. Un jeu de cartes «Lignes directrices pour la qualité de la médecine» peut être commandé sous l'adresse e-mail suivante: fmhqualitaet@ hin.ch 